\title{
Abl deregulates Cdk5 kinase activity and subcellular localization in Drosophila neurodegeneration
}

\author{
$\mathrm{H} \operatorname{Lin}^{1,2}, \mathrm{~T}-\mathrm{Y} \operatorname{Lin}^{1,2}$ and J-L Juang ${ }^{\star, 1}$
}

Although Abl functions in mature neurons, work to date has not addressed Abl's role on Cdk5 in neurodegeneration. We found that $\beta$-amyloid (AB42) initiated Abl kinase activity and that blockade of Abl kinase rescued both Drosophila and mammalian neuronal cells from cell death. We also found activated Abl kinase to be necessary for the binding, activation, and translocalization of Cdk5 in Drosophila neuronal cells. Conversion of p35 into p25 was not observed in A 342 -triggered Drosophila neurodegeneration, suggesting that Cdk5 activation and protein translocalization can be p25-independent. Our genetic studies also showed that abl mutations repressed A 342 -induced Cdk5 activity and neurodegeneration in Drosophila eyes. Although A 342 induced conversion of p35 to p25 in mammalian cells, it did not sufficiently induce Cdk5 activation when c-Abl kinase activity was suppressed. Therefore, we propose that Abl and p35/p25 cooperate in promoting Cdk5-pY15, which deregulates Cdk5 activity and subcellular localization in A $\beta 42$-triggered neurodegeneration.

Cell Death and Differentiation (2007) 14, 607-615. doi:10.1038/sj.cdd.4402033; published online 25 August 2006

Alzheimer's disease $(A D)$, the most frequently diagnosed neurodegenerative disease, is characterized histologically by two hallmark features: senile plaques, which are extracellular deposits of $\beta$-amyloid(A $\beta 42)$, and intracellular neurofibrillary tangles of aggregated hyperphosphorylated tau protein. ${ }^{1}$ The accumulation of $\mathrm{A} \beta 42$ has been found to precede the pathological neurodegenerational changes, ${ }^{2}$ and the inhibition of $A \beta 42$ accumulation by amyloid vaccination can effectively prevent the pathogenesis in mammalian $A D$ animals. ${ }^{3}$ Less clear is the downstream protein targets of A $\beta 42$ toxicity in neurons. Thus, study of the A $\beta 42$-induced signaling cascade is of interest because it may provide clues for designing therapeutic strategies for the treatment of $A D$.

AD brain samples show elevated cdk5 activity, ${ }^{4}$ and deregulated $\mathrm{Cdk} 5$ has been associated with the tau hyperphosphorylation, a state leading to $A D .^{5}$ Like other Cdk members, Cdk5 needs to bind with its regulatory partners to activate kinase activity. One of Cdk5's activating partners, p35, is expressed primarily in postmitotic neurons. ${ }^{6} \mathrm{Cdk} 5$ also appears to be deregulated by its association with p25, a calpain digestion product of $\mathrm{p} 35$ in AD neurons. ${ }^{7}$ The Cdk5/ p25 complex is thought to hyperphosphorylate tau and reduce tau's association with microtubules, resulting in neuronal apoptosis. ${ }^{8}$ Recently, c-Abl has been reported to phosphorylate and activate Cdk5 through the adaptor protein Cables during brain development. ${ }^{9}$ The integration of $\mathrm{Abl}$ and Cdk5 into the same biological pathway suggests they have similar roles in neuronal cytoskeletal regulation and axon guidance.

The Abl kinase, apparently evolutionarily conserved from flies to humans, is involved in the development of the nervous system. ${ }^{10}$ Based on genetic studies of mice ${ }^{11}$ and Drosophi$l a,{ }^{12} a b /$ mutations may lead to neuronal defects. Abl kinase has also been implicated in the regulation of apoptosis. ${ }^{13}$ Interestingly, the cytotoxicity of $A \beta 42$ is considered a crucial stress factor causing neuronal cells to enter apoptosis in the mammalian $A D$ brain. ${ }^{14}$ Therefore, it is worthwhile to investigate possible involvement of $\mathrm{Abl}$ kinase in the progression of neurodegeneration. A growing body of evidence has suggested that AD-related molecular factors are highly conserved in Drosophila, including the amyloid precursor protein-like (Appl), $\gamma$-secretase, presenilin, Cdk5, and tau. ${ }^{15}$ Several groups ${ }^{16-19}$ have attempted to generate Drosophila $A D$ model by expressing a human $A \beta 42$ or $A \beta 40$ in compound eyes and CNS. Similar to the findings in mammals, studies in flies have found neuronal lost phenotype is correlated with the A $\beta 42$ (but not $A$ B40) expression and enhanced with age. Thus, Drosophila might be used as an alternative means of studying the molecular mechanisms in such human neurodegenerative diseases as $\mathrm{AD} .^{20}$

This study used a Drosophila model system to explore the involvement of $\mathrm{Abl}$ in the regulation of Cdk5 during the amyloid-initiated neurodegeneration. We first show that the suppression of Abl expression or kinase activity diminished the activation of Cdk5 triggered by $\mathrm{A} \beta 42$ and reduced Drosophila neuronal cell death. Our genetic studies also supported the hypothesis that $\mathrm{Abl}$ is needed to induce the Cdk5 kinase activity that causes neurodegeneration. In addition, A $\beta 42$-induced neurodegeneration in mammalian cells was rescued by an Abl kinase inhibitor, STI571 (Gleevec, Imatinib). Cleavage of p35 to p25 by A $\beta 42$ did not seem to sufficiently induce Cdk5 activation when c-Abl kinase activity was suppressed. Our findings suggest that the role of Abl on Cdk5 is conserved from fly to human in the amyloid-induced neurodegeneration.

${ }^{1}$ Division of Molecular and Genomic Medicine, National Health Research Institutes, Zhunan Town, Miaoli County, Taiwan

${ }^{*}$ Corresponding author: J-L Juang, Division of Molecular and Genomic Medicine, National Health Research Institutes, 35, Keyan Road, Zhunan Town, Miaoli County 350, Taiwan. Tel: 886-37-246-166 ext.35308; Fax: 886-37-586-459; E-mail: juang@ @nhri.org.tw

${ }^{2}$ These authors contributed equally to this work.

Keywords: Abl; Cdk5; Drosophila; Alzheimer's disease; neurodegeneration

Abbreviations: $\mathrm{AD}$, Alzheimer's disease; $\mathrm{A} \beta$, amyloid $\beta$ peptide

Received 12.12.05; revised 22.6.06; accepted 24.7.06; Edited by E Baehrecke; published online 25.8.06 


\section{Results}

A/42 activates Abl kinase activity for Drosophila neuronal death. To assess the use of Drosophila model to measure $A \beta 42$ neuronal toxicity, we found treatment with A 342 caused dose-dependent cell death in Drosophila neuronal cells, BG2-c2 ${ }^{21}$ (Figure 1a). In contrast, treatment with reversed $\mathrm{A} \beta 42$ peptide did not show notable neuronal toxicity, suggesting that the effect we observed was specific to $A \beta 42$ (data not shown). Intriguingly, inhibiting the expression of Abl by double-stranded RNA (dsRNA) clearly prevented $A \beta 42$-induced neuronal death (Figure $1 \mathrm{~b}$ ). The dsRNA-abl suppressed the Abl expression in a dosedependent manner, and the efficacy of this inhibitory effect was unaffected by treatment with $\mathrm{A} \beta 42$ (Figure 1c). No detectable change in viability was observed after treatment with the control, dsRNA-EGFP (data not shown).

To investigate whether Abl kinase activity could be modulated by $A \beta 42$ in neurons, BG2-c2 cells were treated with $\mathrm{A} \beta 42$ and $\mathrm{Abl}$ activity was indirectly measured by monitoring the tyrosine-phosphorylation level of Ena. ${ }^{22}$ Notably, A $\beta 42$ elevated Ena phosphorylation levels, but STI571 lowered them (Figure 1d), a finding suggests $A \beta 42$ stimulated Abl kinase activity. To determine whether activated $\mathrm{Abl}$ is necessary for $\mathrm{A} \beta 42$-induced neuronal death, $\mathrm{Abl}$ kinase activity was suppressed by STI571 in A $\beta 42$-treated neurons and cell survival was monitored. Treatment with STI571 halted A $\beta 42$-induced neuronal death (Figure 1e). As STI571 also hit other kinases (e.g. PDGFR and c-Kit), ${ }^{23}$ we cannot rule out the possible involvement of other non-Abl kinases.

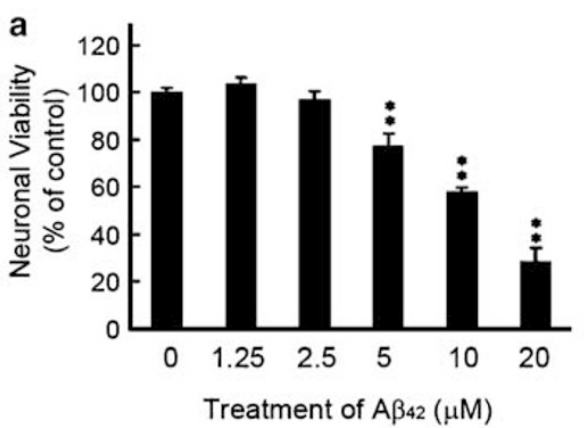

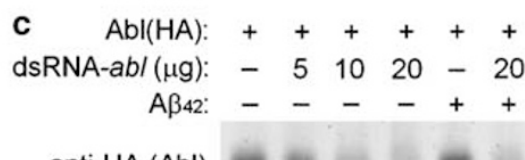
$\operatorname{anti-HA}(\mathrm{Abl})$

anti-Cdk5

anti-actin

d

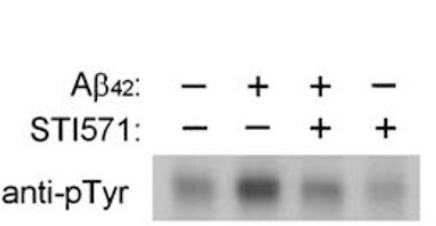

anti-Ena

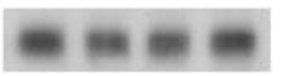

Ena IP b

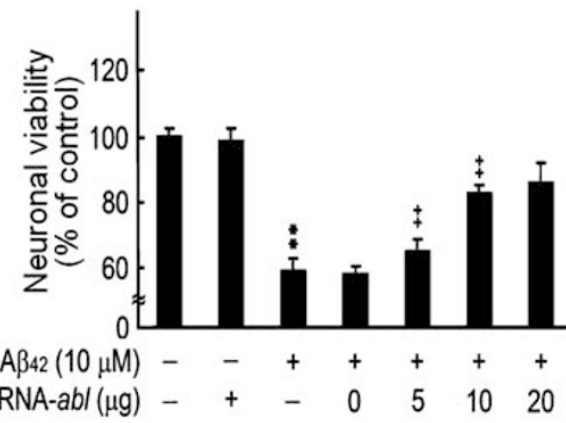

dsRNA-EGFP $(\mu \mathrm{g}):-\quad 51020$

anti-HA (Abl)

anti-actin

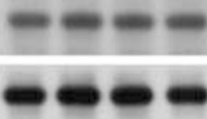

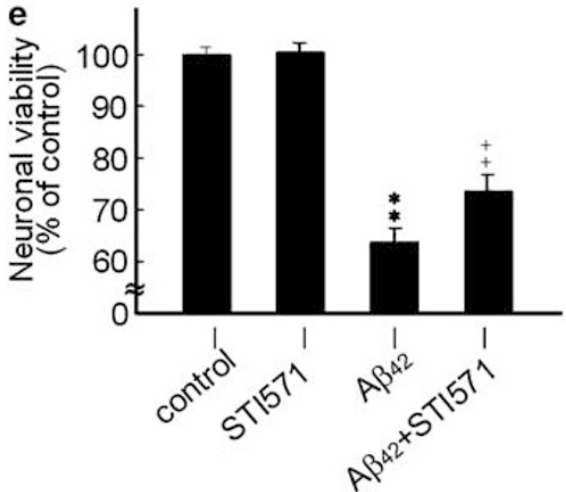

Figure 1 Suppression of Abl kinase activity or Abl expression rescues $A \beta 42$-induced neuronal death in Drosophila. (a) Treatment of $A \beta 42$ for $24 \mathrm{~h}$ caused a dosedependent decrease of BG2-c2 cell viability. (b) Effect of increased dosages of dsRNA-ab/ on A $\beta 42$-induced neuronal death. DsRNAs were introduced by transfection 2 days before treating with $\mathrm{A} \beta 42$ for $24 \mathrm{~h}$. Viability values of control group were adjusted to $100 \%(n=4) ;{ }^{* *}: P<0.01$ versus control group; ${ }^{+}{ }^{+}: P<0.01$ versus $\mathrm{A} \beta 42$ group. (c) Controls of dsRNA-abl. The efficiency and specificity of dsRNA-abl (0-20 $\left.\mu \mathrm{g} / 2 \times 10^{6} \mathrm{cells}\right)$ were evaluated by monitoring the expressions of $\mathrm{HA}$-tagged Abl, Cdk5, and actin (left panel). DsRNA-EGFP was used as nonspecific control (right panel). (d) A $\beta 42$ activated Abl kinase activity. BG2-c2 cells were treated with A $\beta 42$ (2 $\mu \mathrm{M}$ ) and/or an Abl kinase inhibitor (STI571, 20 $\mu \mathrm{M}$ ) for $1 \mathrm{~h}$ to investigate the effect on overexpressed-Ena phosphorylation by Abl. (e) A $\beta 42$-induced neuronal death was repressed by STI571 in Drosophila neuronal cells. Neuronal viability was determined by MTT assays after $24 \mathrm{~h} \mathrm{~A} \beta 42$ treatment 
However, our data clearly suggest that $\mathrm{Abl}$ is an important cellular factor involved in A $\beta 42$-triggered Drosophila neuronal death.

Activated Abl kinase is essential for Abl-Cdk5 interaction and Cdk5 activation. We speculated that $A b$ might be an upstream regulator for Cdk5 activation in response to $A \beta 42$ stimulus. To explore this idea, we first investigated whether $\mathrm{A} \beta 42$-triggered $\mathrm{Abl}$ activation could affect the physical association of Abl and Cdk5. To determine the interaction between $\mathrm{Abl}$ and Cdk5, endogenous Cdk5 was co-immunoprecipitated with exogenous $\mathrm{HA}$-tagged $\mathrm{Abl}$ in BG2-c2 cells. Treatment with $\mathrm{A} \beta 42$ facilitated the AblCdk5 interaction (lane 4 in Figure $2 a$ and $b$ ). Moreover, when Abl activity was suppressed, the Abl-Cdk5 association in cells was dramatically reduced (lane 5 in Figure $2 a$ and b). We also generated antibody against Drosophila Abl to determine whether the endogenous $\mathrm{Abl}$ associates with the endogenous Cdk5, and found the similar results (Figure $2 \mathrm{c}$ ).

We wanted to test whether Cdk5 was activated in the AblCdk5 complex. Using histone $\mathrm{H} 1$ as substrate, we immunoprecipitated Cdk5 from the cell lysate for in vitro kinase assay. Drosophila Cdk5 kinase activity was higher after treatment with $A \beta 42$ than in the untreated controls (Figure $2 b$ ). The elevated Cdk5 activity was corresponded with the activated Abl because STI571's repression of Abl activity not only abolished the Abl-Cdk5 interaction but it also sharply diminished Cdk5 kinase activity (Figure 2b). Moreover, using other Abl kinase regulating agents, doxorubicin (DX) to
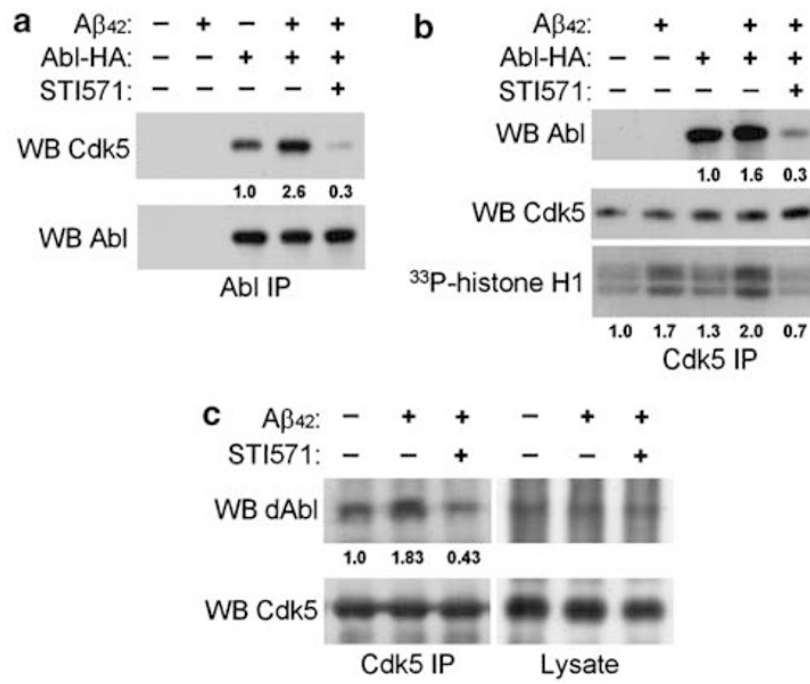

Figure $2 \mathrm{~A} \beta 342$-triggered $\mathrm{Abl}$ kinase activity is required for $\mathrm{Abl}-\mathrm{Cdk} 5$ interaction and Cdk5 activation in Drosophila neuronal cells. (a) Abl-Cdk5 interaction was enhanced by activation of Abl activity. Neuronal cells were transfected with Abl-HA 2 days before treatment with $\mathrm{A} \beta 42(2 \mu \mathrm{M})$ or STI571 $(20 \mu \mathrm{M})$ for $1 \mathrm{~h}$. Cell lysates were subjected to anti-HA immunoprecipitation followed by both anti-Cdk5 and anti-HA immunoblotting. (b) Elevation of Cdk5 kinase activity required Abl kinase activity. Cdk5 was immunoprecipitated for in vitro kinase assays using histone $\mathrm{H} 1$ as substrate. (c) Coimmunoprecipitation of endogenous Abl and Cdk5. Total cell lysates $(2 \mathrm{mg})$ were immunoprecipitated with anti-Cdk5 antibodies. Lysates $(50 \mu \mathrm{g}$; right panel), immunoprecipitates (left panel) were resolved by SDS-PAGE and immunoblotted with indicated antibodies activate and erbstatin analog (EA) to suppress Abl kinase, we also demonstrated that $A b l$ kinase activity was required for the modulation of Cdk5 kinase (data not shown). Taken together, these results suggest that Abl kinase activity is essential for Abl-Cdk5 interaction and Cdk5 activation in A 342 -triggered neurodegeneration.

In vivo role of $\mathrm{Abl}$ in activating Cdk5 kinase for $\mathrm{A} \beta 42-$ triggered neurodegeneration. To further validate our findings in vivo, we used a transgenic fly model, which ectopically expresses $\mathrm{A} \beta 42$ in developing compound eyes. ${ }^{16-19}$ By crossing the UAS-A $\beta 42$ transgenic lines to the eye-specific gmr-GAL4 driver in either wild-type or abl mutant backgrounds $\left(a b l^{1} / D f(3 L) s t-j 7, K I\right),{ }^{24}$ we clarified the role of $\mathrm{Abl}$ on $\mathrm{A} \beta 42$-triggered neuronal defects based on the severity of adult eye phenotypes. In the wild-type background, the adult flies in which $\mathrm{A} \beta 42$ was expressed showed facet disorder (fused ommatidium) and bristle loss in compound eyes, whereas the control gmr-GAL4 did not (Figure $3 a$ and $b$ ). Notably, the $A \beta 42$-induced phenotypes were suppressed by the mutation of $a b l$ (Figure $3 \mathrm{~d}$ ), indicating that $\mathrm{Abl}$ may be an important contributor to A 342 -induced neurodegeneration in Drosophila compound eyes.
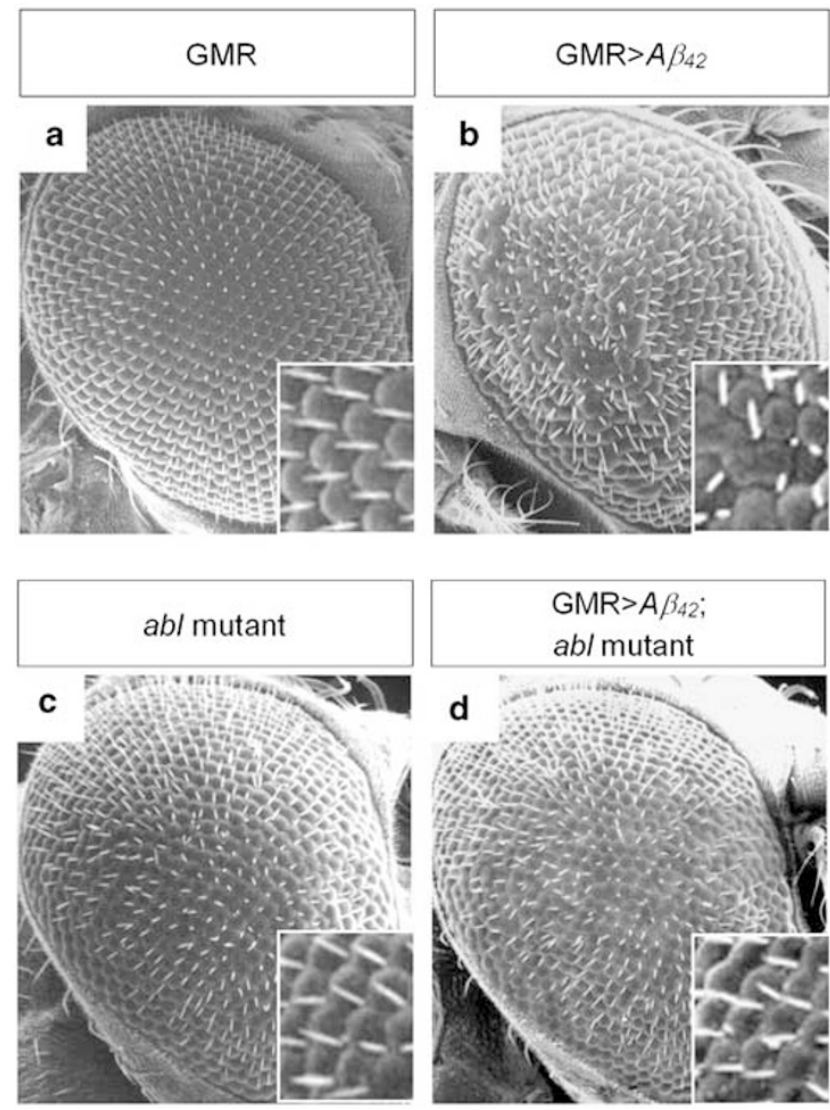

Figure 3 Ectopic expression of $\mathrm{A} \beta 42$ induces rough eye phenotypes in Drosophila compound eyes, which are repressed in abl mutant flies. A $\beta 42$ was ectopically expressed by the eye-specific gmr-GAL4 driver in wild-type and $a b l$ mutant flies. (a-d) Adult eye phenotypes were examined by scanning electron microscope. Magnification is $\times 200(\times 1000$ for the inset) 

kinase activity. ${ }^{9}$ To understand the role of Drosophila Cdk5 on this event, we investigated whether the phosphorylation of Cdk5-Y15 could be correlated with the pathological activation of Cdk5 in neuronal cells. Cdk5-Y15 phosphorylation (Cdk5pY15) levels in cells were examined over time following treatment with $\mathrm{A} \beta 42$. The Cdk5-pY15 in the immunoprecipitates was observed by immunoblotting with an anti-phospho antibody specific for Cdk5-pY15. Within 60 min, A $\beta 42$ brought about an elevation of Cdk5-pY15, which paralleled an increase in Cdk5 activity (Figure 4a). We also transfected the kinase-inactive $\mathrm{Cdk} 5$ into the $\mathrm{A} \beta 42$-stimulated neuronal cells, and found that the Cdk5-pY15 signal and the neuronal death were diminished (data not shown). Once cells had been treated with $\mathrm{A} \beta 42$, Abl was able to regulate Cdk5 activity (Figure 2), leading us to hypothesize that Abl may be Cdk5Y15 kinase. To test this hypothesis, we repressed Abl activity with STI571 in A $\beta 42$-treated cells and found that both the Cdk5-pY15 and kinase activity were diminished (Figure 4b). Moreover, we transfected dsRNA-abl into cells to reduce Abl expression 2 days before $\mathrm{A} \beta 42$ treatment. Consistent with the results with STI571, these cells exhibited a reduction in
Y15 phosphorylation of Cdk5 by the Abl upregulates its

Cdk5-pY15 and kinase activity (Figure 4c), indicating that Abl is crucial in the activation of Cdk5 induced by $\mathrm{A} \beta 42$.

This finding was further substantiated in vivo by immunoprecipitating endogenous Cdk5 from w.t. and $a b /$ mutant adult fly heads to compare their Cdk5-pY15 levels and Cdk5 activities. The Cdk5-pY15 signal and kinase activity were both elevated in flies overexpressing $A \beta 42$ than in the control flies (Figure 4d, lanes 1, 2). In contrast, Cdk5-pY15 signal and Cdk5 activity was reduced in $a b l$ mutant flies overexpressing A $\beta 42$ (Figure $4 d$, lanes 3,4 ). In summary, Abl is involved in A 342 -induced neuronal apoptosis via the regulation of Cdk5.

p35 is not cleaved into p25 in Drosophila neurodegeneration model. p35 is conserved in Drosophila and functions to modulate Cdk5 activity during neurite outgrowth. $^{25}$ In AD brain, mammalian p35 has been cleaved into p25 by calpain to deregulate Cdk5. ${ }^{26}$ Although putative calpains are also present in Drosophila, ${ }^{27}$ the consensus calpain cleavage site is absent in Drosophila p35 (Dp35) (Figure 5a). Therefore, it is unclear whether Dp35

a
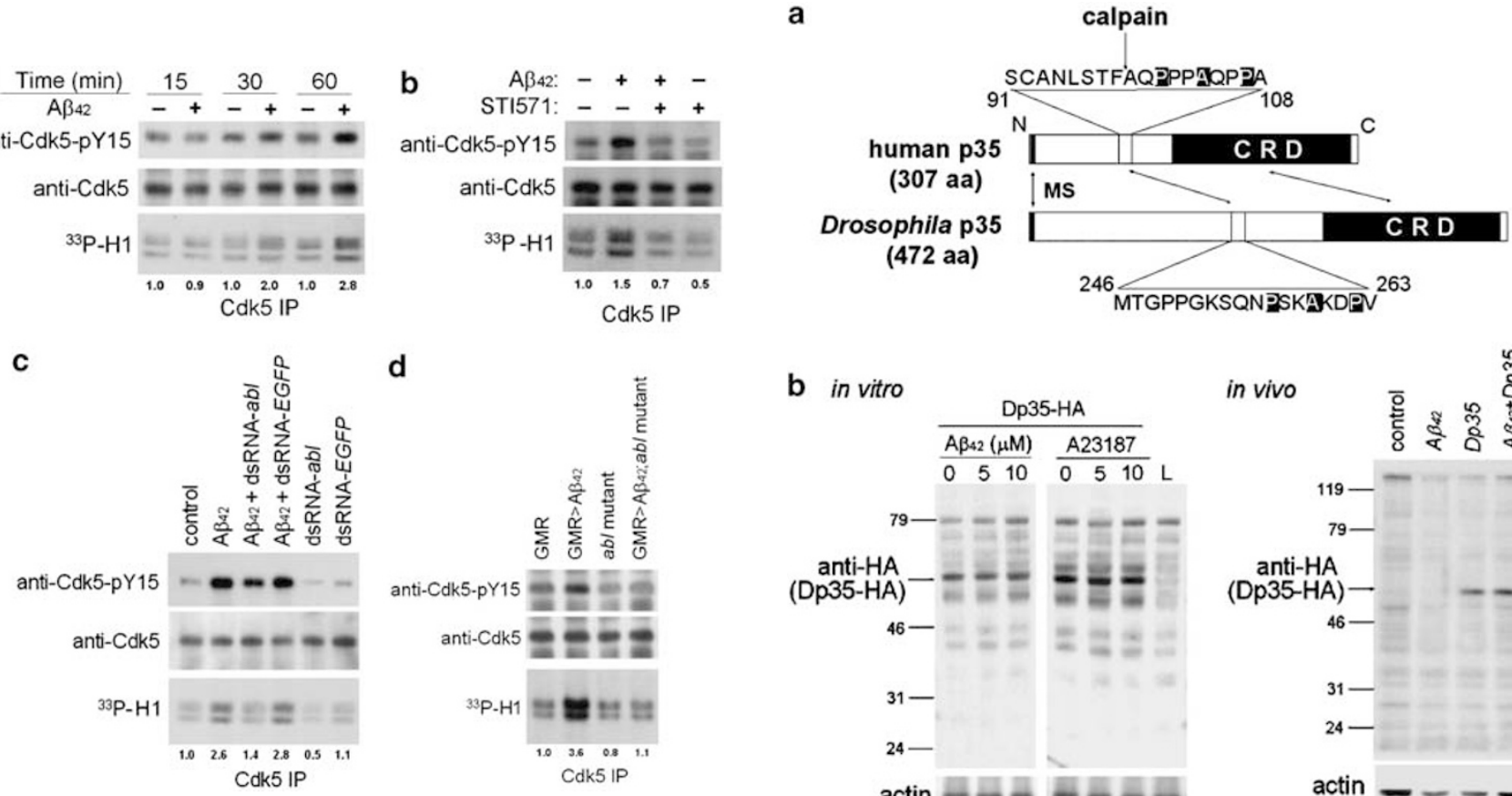

d

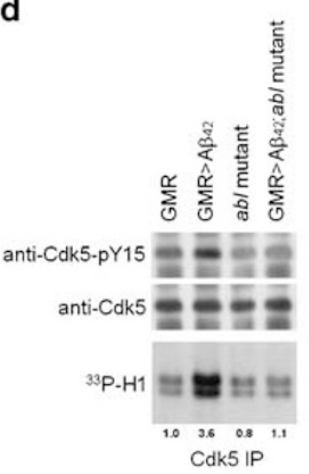

Figure $4 \quad C d k 5-Y 15$ phosphorylation by $A b l$ is crucial for $A \beta 42$-induced Drosophila neurodegeneration. (a) Time-dependent elevation of Cdk5-pY15 and Cdk5 kinase activity following $A \beta 42$ stimulus. Drosophila neuronal cells were treated with $A \beta 42(2 \mu \mathrm{M})$ for $15-60$ min to investigate its effect on Cdk5-pY15 and Cdk5 kinase activity over the time-course. (b) Inhibition of Abl kinase reduced A $\beta 42$ induced Cdk5-pY15 and Cdk5 kinase activity. STI571 (20 $\mu \mathrm{M})$ and/or A $\beta 42(2 \mu \mathrm{M})$ were added simultaneously to neuronal cells for $60 \mathrm{~min}$. (c) Suppression of $A b l$ protein expression reduced $\mathrm{A} \beta 42$-induced Cdk5 kinase activity. Cdk5-pY15 was markedly suppressed (thought not entirely) by transfection of dsRNA-abl into BG2c2 cells $\left(20 \mu \mathrm{g} / 2 \times 10^{6} \mathrm{cells}\right)$. DsRNA-EGFP served as a nonspecific control. Comparable levels of immunoprecipitated Cdk5 were detected with anti-Cdk5 antibody. (d) Abl modulation of Cdk5-pY15 and Cdk5 kinase activity in vivo. Cdk5 protein from different strands of fly heads was examined for its $\mathrm{Y} 15$ phosphorylation and kinase activity. The numbers below the figures represent the relative Cdk5 kinase activity

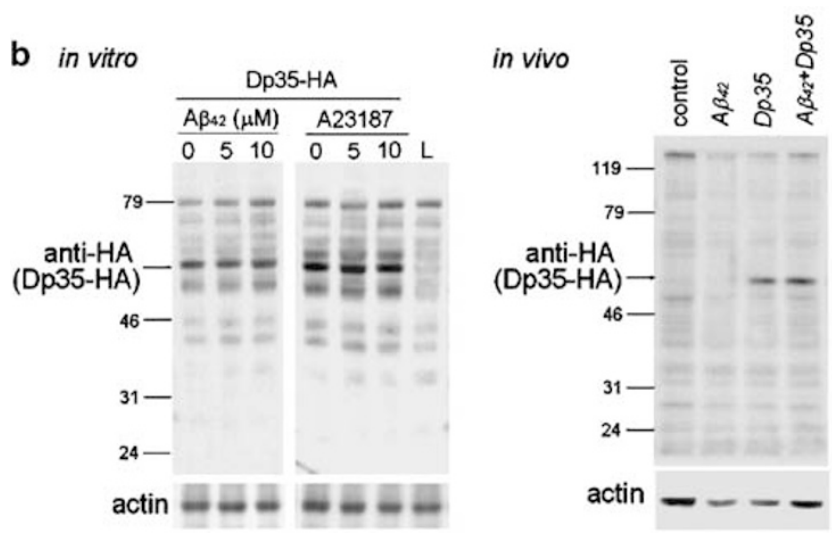

Figure 5 Cdk5 activation is p25-independent in A $\beta 42$-stimulated Drosophila neurons. (a) Structural conservation of p35 between human and Drosophila. Putative calpain digesting site is shown in human p35 sequence but the consensus sequence is not conserved (highlighted characters show the conserved amino acids) in Drosophila. MS, myristoylation site. CRD, Cdk5 regulatory domain. (b) The uncleavable feature of Drosophila p35 in A $\beta 42$-stimulated neuronal cells. Left panel, 2 days after transfection of carboxyl-terminal HA-tagged Dp35, Drosophila neuronal cells were treated with 5 or $10 \mu \mathrm{M} \mathrm{A} \beta 42$ or calcium ionophore (A23187 with $5 \mu \mathrm{M}$ $\mathrm{CaCl}_{2}$ ) for an additional $24 \mathrm{~h}$. Cell lysates were immunoblotted with anti-HA and antiactin antibodies to examine the Dp35 cleavage and protein loading respectively. Right panel, adult fly heads ectopically expressing A $\beta 42$ and/or Dp35-HA (driven by gmr-GAL4) were lysed and immunoblotted with anti-HA and anti-actin antibodies. Consistently, these results revealed no detectable cleavage or reduction of Dp35$\mathrm{HA}$ protein $(\sim 53 \mathrm{kDa})$ in either the cell culture or transgenic systems 
will convert into p25 when stimulated by $\mathrm{A} \beta 42$. To investigate this possibility, we transfected HA-tagged Dp35 into cells before treating them with $\mathrm{A} \beta 42$ or calcium ionophore. Intriguingly, we did not observe any noticeable cleavage or reduction of Dp35 levels in the lysates (Figure 5b, left panel). To analyze the Dp35 cleavage in vivo, we generated transgenic flies coexpressing Dp35 and $\mathrm{A} \beta 42$ in eyes by a gmr-GAL4 driver. We also observed no visible cleavage or reduction of the Dp35 in the $A \beta 42$ transgenic flies (Figure $5 b$, right panel). These results suggest that Dp25 or other truncated forms of Dp35 may not be required for the $\mathrm{A} \beta 42$ induced Drosophila neurodegeneration. However, the absence of Dp35 cleavage in neurodegenerative flies does not necessarily imply that p35 is dispensable for the regulation of Cdk5 in Drosophila neurodegeneration. To test this idea, dsRNA-Dp35 was transfected before the cells were stimulated with $A \beta 42$. The Dp35 protein levels were repressed by dsRNA-Dp35 in a dose-dependent manner (Figure 6a, left panel). Although Cdk5-Y15 was still phosphorylated in response to $\mathrm{A} \beta 42$, the Cdk5 activity was abolished after Dp35 was depleted from cells (Figure 6a, right panel). This result suggests that the induction of Cdk5 by $\mathrm{Abl}$ still requires the involvement of $\mathrm{p} 35$.

To address the cooperative role of $\mathrm{Abl}$ and p35 in neurodegeneration, we conducted a genetic interaction

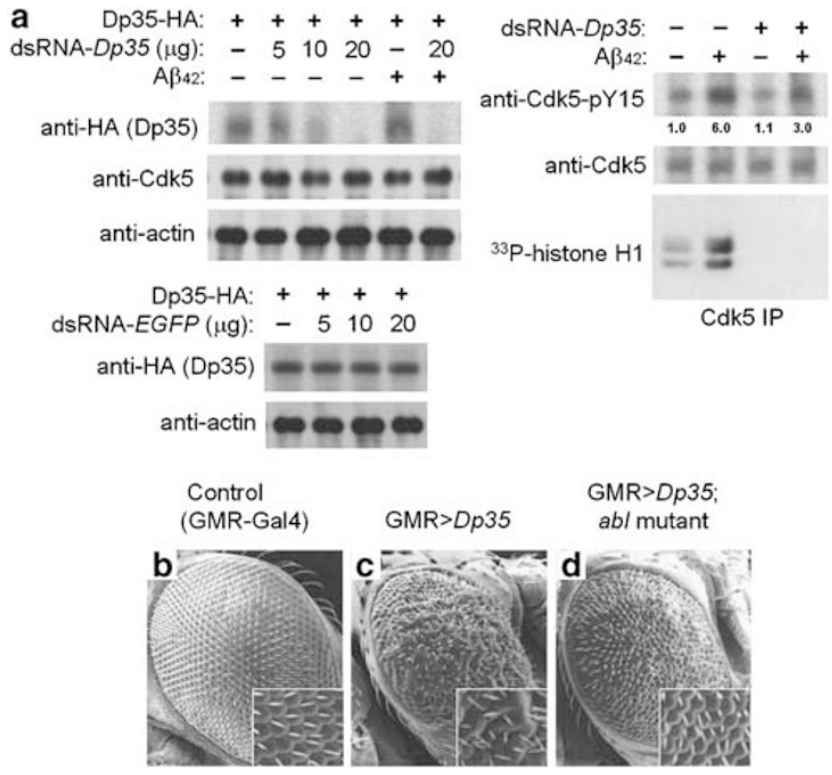

Figure 6 Ectopic expression of Dp35 induces Cdk5 activation and neurodegeneration in Drosophila eye discs, which can be suppressed by abl mutant. (a) Repression of Dp35 expression totally abrogated A $\beta 42$-induced Cdk5 activation. Left panel, the silencing efficiency and specificity of dsRNA-Dp35 $\left(0-20 \mu \mathrm{g} / 2 \times 10^{6}\right.$ cells) were evaluated by monitoring HA tagged Dp35, Cdk5, and actin. No visible interference of dsRNA efficiency by $A \beta 42$ was noted (lane 6). DsRNA-EGFP served as a nonspecific control. Right panel, Y15 phosphorylation level and kinase activity of Cdk5 were determined whereas Dp35 was targeted by dsRNA in cells treated with A $\beta 42$. DsRNA-Dp35 was transfected into neuronal cells 2 days before $A \beta 42$ treatment. After $1-\mathrm{h} A \beta 42$ treatment, cells were lysed and immunoprecipitated with anti-Cdk5 antibody to study Cdk5-pY15 and kinase activity. The Cdk5-pY15 signal was largely retained but its kinase activity was dramatically abolished. (b-d) Scanning electron microscope images of adult transgenic compound eyes phenotypes. Magnification is $\times 200(\times 1000$ for the inset) assay. Overexpression of Dp35 in the eyes by a gmr-GAL4 driver resulted in severe adult rough eye phenotypes (Figure 6c). Consistent with the results from $A \beta 42$-induced eye phenotypes, abl mutant suppressed the Dp35-induced rough eye phenotype (Figure $6 \mathrm{~d}$ ). Because overexpression of Dp35 has been shown to induce Cdk5 hyperactivation, ${ }^{25}$ the finding of abl mutant suppressed Dp35-induced phenotypes further substantiates the role of $\mathrm{Abl}$ in neurodegeneration.

Cdk5-Y15 phosphorylation by $\mathrm{Abl}$ is required for $\mathrm{A} \beta 42-$ induced Cdk5 subcellular translocalization. It was postulated that the amino-terminal myristoylation sequence of mammalian p35 is the key domain anchoring Cdk5-p35 complex to the cell membrane. If so, removal of the p35 amino-terminus, producing p25, would sever the complex's association with the cell membrane and thus translocalize Cdk5 to cytosol. ${ }^{7}$ Intriguingly, although the cleavage of p35 into p25 may be absent in fly neurodegenerative neurons, the myristoylation site is conserved in Dp35 (Figure 5a). The unique sequence feature of Dp35 suggests that Drosophila Cdk5-p35 complex may be resident on the cell membrane and cannot be translocalized to the cytosol. To explore this speculation, endogenous Cdk5 localization was examined by immunostaining. Without the stimulus of $A \beta 42$, the $\mathrm{Cdk} 5$ was mainly expressed on the cell membrane with less in the cytosolic region (Figure $7 \mathrm{a}$ ), which is similar to findings in mammalian studies. ${ }^{28}$ Unexpectedly, $1 \mathrm{~h}$ after $\mathrm{A} \beta 42$ stimulus, Cdk5 was markedly translocalized from cell membrane to the perinuclear cytoplasm (Figure 7b), which suggests a p25independent mechanism for Cdk5 translocalization. By treating cells with $\mathrm{BL}-1$ (Cdk5 kinase inhibitor) simultaneously with $A \beta 42$ to block the Cdk5 activation, the translocalization of Cdk5 to perinuclear cytoplasm was abrogated (data not shown), indicating it is activitydependent regulation of Cdk5. These results suggest that the accumulation of Drosophila Cdk5 within the cells is affected by the kinase activity of Cdk5.

As phosphorylation of Cdk5-Y15 correlates with Cdk5 activity, we investigated whether the Cdk5-pY15 is required for directing Cdk5 subcellular localization. Without the $A \beta 42$ stimulus, $\mathrm{Cdk} 5^{\mathrm{Y} 15 \mathrm{~F}}$ was primarily localized to the cell membrane similar to the wild-type Cdk5 (Figure 7c). However, treatment of $\mathrm{A} \beta 42$ did not noticeably translocalize $\mathrm{Cdk} 5^{\mathrm{Y} 15 \mathrm{~F}}$ to the perinuclear cytoplasm (Figure7d, arrow), in contrast to the untransfected cells (Figure $7 d$, arrowhead). Thus, the translocation of Cdk5 may be modulated by the Abl phosphorylation of Cdk5-Y15. To test this idea, we suppressed Abl activity before stimulating cells with $\mathrm{A} \beta 42$ and immunostained both the Abl-HA and the endogenous Cdk5. The Abl and Cdk5 were colocalized to the plasma membrane and cytoplasm in control cells (Figure 7g, j, m). Upon stimulation with $A \beta 42$, the colocalization of $\mathrm{Cdk} 5$ with $\mathrm{Abl}$ in the perinuclear cytoplasm was increased (Figure $7 \mathrm{~h}, \mathrm{k}, \mathrm{n}$ ). However, such protein translocalization was markedly repressed when Abl activity was blocked (Figure 7i, I, o, arrow). We also transfected dsRNA-abl into neuronal cells and found the similar results (data not shown). These results accord well with the role of Abl on modulation of Cdk5 in neurodegeneration. 

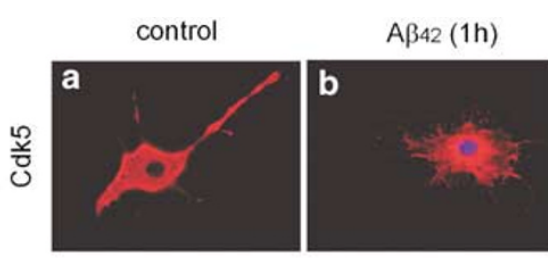

control
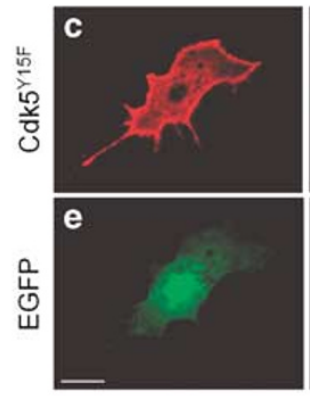

control
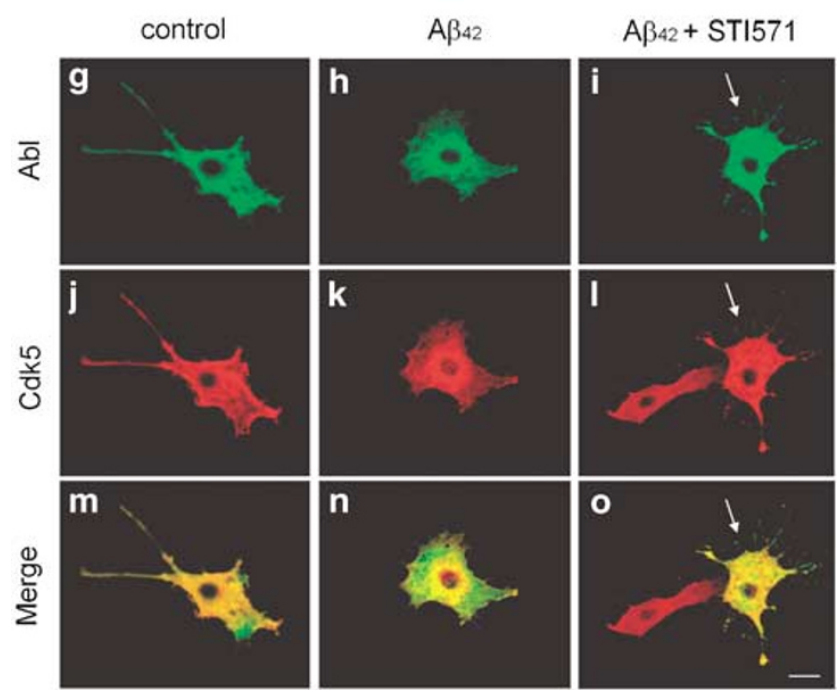

Figure $7 \mathrm{~A} \beta 42$ affects $\mathrm{Cdk5}$ intraneuronal translocalization by a $\mathrm{p} 25$ independent, Abl-associated phosphorylation of Cdk5-Y15 in Drosophila neuronal cells. (a-b) Confocal microscopy analysis of immunostaining of endogenous Cdk5 (TRITC-labeled) in BG2-c2 neuronal cells. Cdk5 translocalized from cell membranes (a) to perinuclear regions (b) following stimulation of cells with $2 \mu \mathrm{M}$ $\mathrm{A} \beta 42$ for $1 \mathrm{~h}$. Nuclei were labeled with DAPI (blue). (c-f) $\mathrm{Cdk} 5^{\mathrm{Y} 15 \mathrm{~F}}$ does not translocalize to perinuclear regions upon $A \beta 42$ stimulation. Cells were cotransfected EGFP plasmid (panels $\mathbf{c}-\mathbf{f}$ ) with $\mathrm{Cdk} 5^{\mathrm{Y} 15 \mathrm{~F}}$ mutant to differentiate the mutant Cdk5-expressing cells (panel $\mathbf{c}$ and arrow in panel $\mathbf{d}$ ) from the nontransfected cells. Stimulation of cells with $A \beta 42$ for $1 \mathrm{~h}$ markedly translocalized endogenous wild-type Cdk5 protein to perinuclear regions (arrowhead in panel d) in contrast to that of exogenous $\mathrm{Cdk} 5^{\mathrm{Y} 15 \mathrm{~F}}$ protein (arrow in panel $\mathbf{d}$ ). $(\mathbf{g}-\mathbf{0})$ The colocalization of $\mathrm{Cdk} 5$ with $\mathrm{Abl}$ in the perinuclear cytoplasm is markedly increased upon stimulation with $A \beta 42$. Cdk5 and $A b l-H A$ were double-immunolabeled to investigate whether they colocalized upon $A \beta 42$ stimulus. Without treatment of $\mathrm{A} \beta 42, \mathrm{Abl}-\mathrm{HA}$ (green) and Cdk5 (red) were colocalized to the cell membrane and cytoplasm (panels $\mathbf{g}, \mathbf{j}$, and $\mathbf{m}$ ). As expected, Abl-HA and Cdk5 colocalized to perinuclear regions in response to $A \beta 42$ stimulus (panels $\mathbf{h}, \mathbf{k}$, and $\mathbf{n} ; 85 \%$ in perinuclear region, $N>50$ ). White arrow indicates an Abl-HA transfected cell exhibited no $\mathrm{Abl}$ or $\mathrm{Cdk5}$ protein translocalization to perinuclear region whereas STI571 and A $\beta 42$ were treated simultaneously to cells (panels i, I, and $\mathbf{0} ; 13 \%$ in perinuclear region, $N>50$ ). Scale bar: $10 \mu \mathrm{m}$

\footnotetext{
A 342-triggered human p35 cleavage into p25 is insufficient to initiate Cdk5 kinase activity in cells deficient of c-Abl kinase activity. To explore the
}

possibility that the role of $\mathrm{Abl}$ in Drosophila neurodegeneration is conserved in mammals, we examined whether c-Abl kinase activity was involved in A $\beta 42$-induced mammalian neuronal apoptosis. C-Abl kinase was suppressed with STI571 in human IMR-32 cells before treatment with $\mathrm{A} \beta 42$, and neuronal viability was determined. Like the results in the Drosophila, the inhibition of c-Abl activity prevented neuronal death in cells (Figure 8a). Moreover, knockdown of c-Abl expression by siRNA also repressed $A \beta 42$-induced neurotoxicity (data not shown). Thus, we wanted to determine whether the conversion of p35 into p25 was sufficient to deregulate Cdk5 activity whereas Abl activation was inhibited. After IMR-32 cells were pretreated with STI571 to block c-Abl activity, they were incubated with $A \beta 42$ to induce the cleavage of human p35. As was found in a previous study, ${ }^{26}$ we found that $A \beta 42$ triggered the cleavage of p35 into p25 and increased Cdk5pY15 and kinase activity (Figure 8b, lane 2). However, in the presence of STI571, both the Cdk5-pY15 and kinase activation were reduced, though the formation of p25 was not obviously affected (Figure 8b, lane 3), suggesting that the cleavage of p35 into p25 was insufficient to fully activate Cdk5 whereas c-Abl activity was suppressed. We speculated that p25 cooperated with c-Abl to help activate Cdk5 kinase because the p25-Cdk5 complex was more stable than that of p35-Cdk5. ${ }^{29}$ To test this hypothesis, a calpain inhibitor was used to inhibit the cleavage of p35 to determine the function of p25 on Cdk5. Interestingly, the blockade of p35 cleavage did not diminish Cdk5-pY15 but the Cdk5 activity was considerably reduced (Figure $8 \mathrm{c}$, lanes 2,3 ), suggesting that the formation of p25 was indeed important for the Cdk5 activation, whereas the phosphorylation of Cdk5-Y15 was independent of p35 cleavage. Moreover, we also found that the cotreatment of cells with Calpeptin and STI571 further reduced the Cdk5 activity (Figure 8c, lane 5), supporting the idea that $\mathrm{Abl}$ and p25 cooperated in deregulating Cdk5 in mammalian cells.

\section{Discussion}

$\mathrm{AD}$ is characterized by the accumulation of $\mathrm{A} \beta$ and the hyperphosphorylation of tau in the neurodegenerative brain. Although Cdk5 is one of kinases that have been implicated in tau phosphorylation, the underlying mechanisms that induce the $\mathrm{A} \beta$ signals to deregulate Cdk5 for the progression of neurodegeneration is not yet clearly understood. By using Drosophila as a model system, our study has provided several lines of evidence supporting the idea that Abl mediates Cdk5 for $\mathrm{A} \beta 42$-triggered neurodegeneration.

The role of Abl in A/42-induced neurodegeneration. Like Cdk5, cellular Abl functions in neural development and its kinase activity and subcellular localization are tightly regulated. ${ }^{10}$ Here, we first show that Abl appears to be essential for A $\beta 42$-triggered Drosophila neurodegeneration both in vivo and in vitro. It is of interest in this regard that Abl may serve as a putative molecular target to stop the progress of neurodegeneration. Interestingly, the anti-leukemic agent Abl kinase inhibitor, STI571, was shown to rescue the A $\beta 42-$ induced neurodegeneration in both Drosophila and 
a

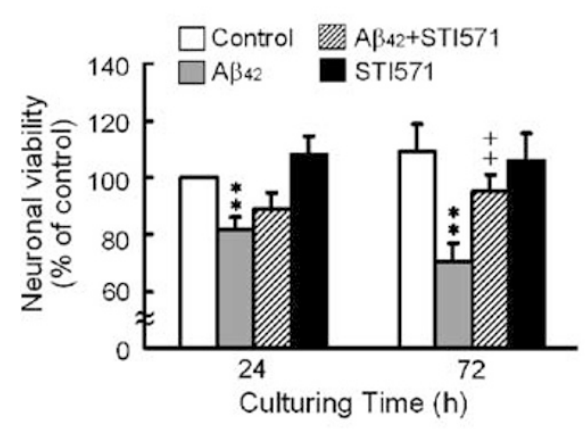

b

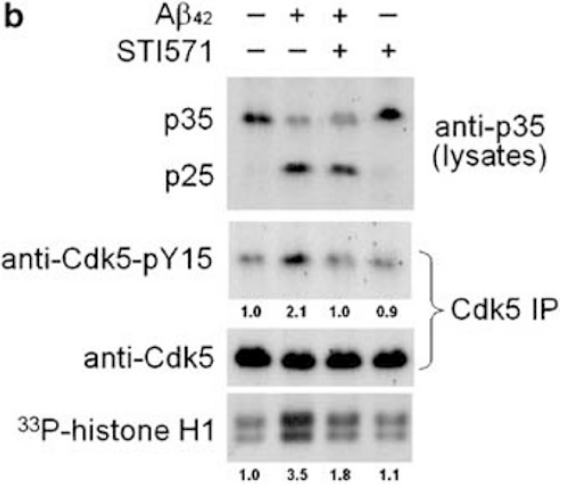

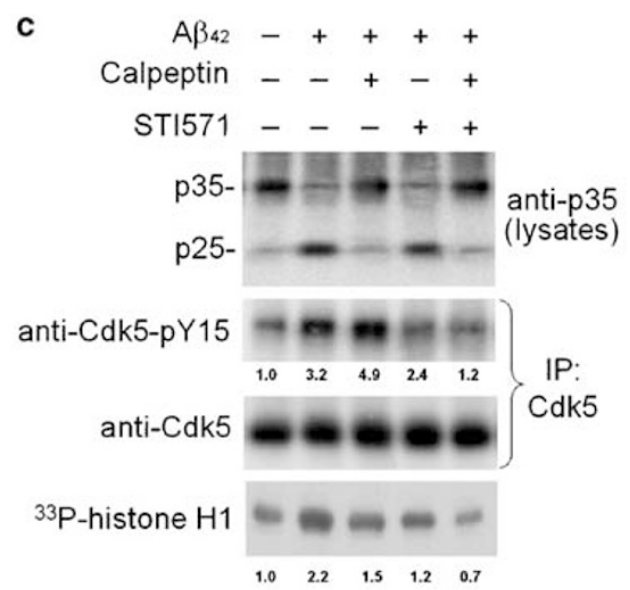

Figure $8 \mathrm{C}$-Abl functions cooperatively with p25 for the induction of Cdk5 activity in A $\beta 42$-induced human neuronal death. (a) STI571 effectively repressed A $\beta 42$-induced IMR-32 neuronal death after $72 \mathrm{~h}$ - treatment of A $\beta 42$. Cells were pretreated for $24 \mathrm{~h}$ with STI571 before stimulation with $\mathrm{A} \beta 42$ for $24 \mathrm{~h}$ or $72 \mathrm{~h}(n=4,24-\mathrm{h}$ control value as $100 \%$; ${ }^{* \star} P<0.01$ versus control group in the same time point; ${ }^{+}+P<0.01$ versus A $\beta 42$ group). (b) Induction of p35 cleavage into p25 by A $\beta 42$ is incapable of initiating Cdk5 kinase activity in cells lacking Abl kinase activity. Human IMR-32 cells were pretreated for $24 \mathrm{~h}$ with STI571 before stimulation with/without A $\beta 42(20 \mu \mathrm{M})$ for $24 \mathrm{~h}$ to examine the effect on Cdk5-pY15 and Cdk5 kinase activity. The cleavage of p35 into p25 by the induction of A $\beta 42$ was not notably affected by the STI571, but Cdk5 kinase activity and Y15 phosphorylation was drastically repressed by STI571 treatment. (c) Cotreatment of calpain inhibitor (Calpeptin) and STI571 further reduced Cdk5 activity comparing to individual treatment (lane 5 versus lane 3 and 4). The numbers below the figures represent the relative Cdk5 kinase activity

mammalian cells. However, STI571 is probably not an ideal reagent for testing this idea in vivo because of its low penetration capability through the blood-brain barrier. ${ }^{30}$ Another previous link between $\mathrm{A} \beta 42$ and $\mathrm{Abl}$ inhibition by STI571 was reported in a recent study by Netzer et al. ${ }^{31} \mathrm{~A} \beta 42$ production was reduced by STI571 in neuronal cultures and in guinea-pig brain. Therefore, it is reasonable to speculate that Abl kinases might affect amyloid signaling at various points including $\mathrm{A} \beta 42$ production.

Deregulation of Cdk5 by Abl in A $\mathbf{A 4 2 - t r i g g e r e d ~ n e u r o - ~}$ degeneration. In light of above findings, we explored whether $\mathrm{Abl}$ acted to deregulate Cdk5 in A $\beta 42$-triggered neurodegeneration. All of our data, including biochemical, genetic, and immunocytochemical studies, supported this view. Specifically, the activated Abl interacted with Cdk5 to potentiate Cdk5 kinase activity through Y15 phosphorylation. The blockade of Abl activation or the reduction of protein expression led to about a $50 \%$ reduction in Cdk5 activity. The proper localization of $\mathrm{Cdk} 5$ is important in regulating its kinase substrates for cytoskeletal dynamics. ${ }^{32}$ Concomitant with elevated Cdk5 kinase activity, Cdk5 was translocalized from the cell membrane to a perinuclear localization. Treatment of cells with STI571 to repress Cdk5-pY15 also inhibited Cdk5 translocalization, a finding that suggests Abl controls Cdk5 shuttling by the phosphorylation of Cdk5-Y15. Furthermore, this hypothesis was further substantiated in vivo (Figures 3 and $4 d$ ). Collectively, our data support the possibility of a novel pathway in which Abl confers regulation on Cdk5 activity and subcellular localization in Drosophila neurodegeneration.

A p25-independent activation and translocalization of Cdk5 kinase in Drosophila neurodegeneration. In the mouse AD brain, p35 was converted to p25 by calpain in cerebral tissue, and suppression of calpain activity effectively repressed p35 cleavage and $\mathrm{A} / 342$-induced neuronal death. $^{26}$ This report imply that p25 may be crucial in augmenting Cdk5 kinase activity in the AD pathogenesis. However, overexpression of p35 has been also found sufficient to initiate Cdk5 kinase activity in both mammalian and Drosophila cells. ${ }^{6,25}$ Our studies also show that, although Abl activates Cdk5, it cannot do so without 
adequate p35 in the cells, demonstrating that like in mammals, p35 is necessary for Cdk5 activity.

One perplexing finding from our fly data is the absence of cleavage of p35 into p25 in neurodegenerative cells, despite the fact that Cdk5 kinase activity was still stimulated by A $\beta 42$. Several recent studies have suggested that p35 plays a critical role in the progression of neurodegeneration. ${ }^{33-35}$ In consideration of the molecular events in the chronic progression of $A D$ pathogenesis, conversion of p35 into p25 may be a progressive and tightly regulated procedure in neurons. Therefore, both p35 and p25 might share a common role in modulating Cdk5 for normal and pathological activities in neurons. Our Cdk5 immunostaining data show an Abldependent mechanism in the regulation of Cdk5 localization by demonstrating that $\mathrm{Cdk} 5$ translocalized to the perinuclear cytoplasm by Abl phosphorylation of Cdk5-Y15 but not the p35 cleavage (Figure 7). Consistent with this finding, two recent reports have suggested that Cdk5 in company with p35 could be transported into nucleus in primary cortical neurons to facilitate apoptosis. ${ }^{36,37}$ Given the fact that Dp35 is not cleaved to Dp25 in Drosophila neurodegeneration, the Drosophila model may be instrumental in testing the function of p35, without the involvement of p25, in deregulating Cdk5 kinase activity.

On the other hand, our mammalian cells study also supports that, by cooperating with c-Abl, p25 may be associated with Cdk5 activation, although we point out that the formation of p25 does not sufficiently trigger Cdk5 activity when Abl activation was blockaded (Figure 8b). The strongest evidence for the requirement of both c-Abl and p25 in facilitating Cdk5 activation and neurodegeneration in $\mathrm{A} \beta 42-$ treated mammalian neurons comes from the parallel blockades of c-Abl activation and p35 cleavage that further abolished endogenous Cdk5 activity. In this regard, it is relevant to conjecture a similar cooperative role of c-Abl and p35 on Cdk5 during the neurodegeneration, presumably by Abl phosphorylation of Cdk5 to stabilize Cdk5-p35 complex. In the present study, we provide the evidence that the induction of amyloid also enhanced Abl-Cdk5 complex. Accordingly, amyloid may facilitate the association of Abl with Cdk5-p35 complex in the degenerative neurons.

In light of ours and others findings, ${ }^{9,26}$ we can postulate a model for $\mathrm{A} \beta 42$-induced neurodegeneration in which the Abl may associate with Cdk5-p35 to modulate Cdk5 kinase activity and subcellular localization, ultimately leading to aberrant cytoskeletal rearrangement and neurodegeneration. Alternatively, Abl may also interact with Cdk5-p25 complex and further deteriorate neurological function. Perhaps neurons provide a flexible and adequate framework for both neuronal plasticity and pathological neurodegeneration in brain.

\footnotetext{
Materials and Methods

Cell culture, DNA constructs, and transfection. Drosophila neuronal cell line, BG2-c2, is a Drosophila melanogaster third-instar larval neuronal cell line cultured in M3 medium (Sigma) plus 10\% FBS (Hyclone), $10 \mu \mathrm{g} / \mathrm{ml}$ insulin (Sigma), and penicillin/streptomycin (BRL-Gibco) in $24.5^{\circ} \mathrm{C}$. IMR-32 (human neuroblastoma) was provided from ATCC (\#CCL-127) and propagated in MEM medium (BRL-Gibco) plus non-essential amino acids (BRL-Gibco) and 10\% FBS (Hyclone).
}

Drosophila constitutive expression vectors for all DNA constructs are pAc5.1 (Invitrogen). cDNA of abl, and ena in pPac vector were subcloned into pAc5.1. Drosophila Cdk5 was obtained from Drosophila EST LD01910 and subcloned into pAc5.1 vector by Not1 and Xba1 cutting sites. Dp35 was obtained from EST $\mathrm{HL} 05519$ and subsequently cloned into HA-tagged pAc5.1 vector. Cdk5 ${ }^{Y 15 F}$ mutant was constructed by PCR-based mutagenesis. Transient transfection of neuronal cells was performed using the liposome-based method with $1 \sim 2 \mu \mathrm{g} \mathrm{DNA} / 10^{5}$ cells. BG2-c2 cells were transfected with dsRNA ( $20 \mu \mathrm{g} / 2 \times 10^{6}$ cells) 2 days before drug treatments for another day.

Cell viability assay. The modified MTT assay (Sigma) was carried out to quantify neuronal cell viability. ${ }^{26}$ The intensities measured by optical density reader (SpectroMAX plus, Molecular Devices) at $570 \mathrm{~nm}$ (background: $630 \mathrm{~nm}$ ) proportionally represented the viable cell numbers. Amyloid was dissolved in water to give solution of $200 \mu \mathrm{m}$ and incubated at $37^{\circ} \mathrm{C}$ for $48 \mathrm{~h}$ before use.

Immunoprecipitation and Western blot analysis. Cell lysate was produced in lysis buffer or extract buffer ${ }^{38}$ for Cdk5 immunoprecipitation. Proteins were analyzed by direct Western blotting $(30 \mu \mathrm{g} / \mathrm{lane})$ or blotting after immunoprecipitation (1-2 mg/immunoprecipitation). Immunoprecipitates were collected by binding to protein G PLUS/Protein A-Agarose (Oncogene Research Products). Antibodies were listed below: anti-Cdk5 antibody (clone DC17, Upstate; sc-750, Santa Cruz), anti-Cdk5-pY15 antibody (sc-12919, Santa Cruz), anti-HA antibody (BAbCO), anti-p35 antibody (sc-820, Santa Cruz) and affinity-purified Ena polyclonal antibody. ${ }^{39} \mathrm{ECL}$ detection reagent was used to detect the immunoreactive proteins. The intensity of the bands was measured by LAS 1000 (Fuji Film). The ratio of intensities between an anti-pY15 antibody image in A $\beta 42$ treatment and an anti-Cdk5 antibody image was used for the analysis of tyrosine phosphorylation of Cdk5.

In vitro Cdk5 kinase assay. Kinase assay was performed by washing immunoprecipitates three times with kinase reaction buffer ( $50 \mathrm{~mm}$ HEPES $(\mathrm{pH} 7.0)$, $10 \mathrm{mM} \mathrm{MgCl}_{2}$, and $1 \mathrm{mM} \mathrm{DTT}$ ). The beads with target proteins were incubated with kinase reaction buffer containing $2 \mu \mathrm{g}$ of substrate (histone $\mathrm{H} 1$, Calbiochem) and $10 \mu \mathrm{Ci}$ of ${ }^{33} \mathrm{P} \gamma$-ATP in a final volume of $40 \mu \mathrm{l}$ at $30^{\circ} \mathrm{C}$ for $30 \mathrm{~min}$.

DsRNA and siRNA assays. DNA fragments including both sense and antisense coding sequences for Drosophila abl (729 bp, CDS: 241-969), EGFP (full-length, $765 \mathrm{bp}$, Invitrogen), and Dp35 (720 bp, CDS: 1-720) were amplified by PCR. PCR products were subcloned into the vector (all cut by BamH1 and blunt-end products were produced), pBluescript (cut by EcoRV, Strategen) and subsequently used as templates to in vitro transcribe by T3/T7 MEGAscript kit (Ambion). Singlestranded RNA were generated and annealed with compensatory strands in $65^{\circ} \mathrm{C}$ for $30 \mathrm{~min}$, followed by $94^{\circ} \mathrm{C}, 5 \mathrm{~min}$ and $72^{\circ} \mathrm{C}, 10 \mathrm{~min}$. DsRNA products were confirmed as a single band by electrophoresis and stored in $-20^{\circ} \mathrm{C}$. To knockdown c-Abl expression in mammalian cells, SH-SY5Y and IMR-32 cells were transfected with cAbl siRNA (Upstate, Abl siRNA SMARTpool ${ }^{\mathrm{TM}}$ ) or control luciferase siRNA for $36 \mathrm{~h}$ before stimulation with $\mathrm{A} \beta 42$ for $48 \mathrm{~h}$. The cell viability was determined by WST-1 reduction assay (Roche). The percentage of viable cells was normalized with that in the control cells.

Immunohistochemistry. Neuronal cells cultured on coverslips (coated by poly-L-lysine, Biochrom AG) were fixed, permeabilized, and blocked as previous described ${ }^{39}$ Primary antibodies (anti-Cdk5, $2 \mu \mathrm{g} / \mathrm{ml}$; anti-HA, 1:500) diluted in $3 \%$ BSA-PBS were incubated with coverslips overnight at $4^{\circ} \mathrm{C}$. Cells were washed in PBS and exposed to FITC or TRITC-conjugated secondary antibodies (affinitypurified goat anti-mouse IgG, 1:2000, Jackson) for $1 \mathrm{~h}$ at RT. After extensive washing, coverslips were mounted in Gel/Mount (Biomeda) and observed by Leica confocal microscopy. Quantification of the subcellular localization of Abl and Cdk5 was analyzed by immunofluorescence microscopy.

Genetics. A cDNA encoding the fragment of human amyloid precursor protein A $\beta 42$, was amplified from a human brain CDNA library by PCR with primer $5^{\prime}$ AAGATGGATGCAGAATTCCGACATGACTCAGGA-3' and $5^{\prime}$-TTAATGATG ATG ATGATGATGCGCTATGACAACACCGCCCACCATGAGTCCAAT-3'. CDNAs of $A \beta 42, D p 35$, cdk5, and $c d k 5^{Y 15 F}$ were separately subcloned into GAL4responsive pUAST vector which was used to microinject for transgenic fly. Embryos of the $\Delta 23 /+$ genotype fly were microinjected and transgenic lines were obtained by standard methods, ${ }^{40}$ and genomic DNA from individual transgenic lines 
were verified by autosequencing. Five independent UAS-A $\beta_{42}$ transgenic strains are generated and three strains were further characterized by crossing with gmrGAL4 flies. The rough eye phenotype of these strains is shown in a range from 72 to 87.2\% penetrant. $a b{ }^{1}$ (a mutant generated by ethyl methanesulfonate mutagenesis) and deficiency $D f(3 L) s t-j 7, K i$ (a small deletion that encompasses the $a b /$ gene) flies ${ }^{26}$ were crossed with transgenic flies to obtain $a b I^{-1}$ background flies with ectopically expressed proteins. The double transgenic flies, which expressed both $A \beta 42$ and Dp35-HA, were also generated by sequent crossing. All ectopically expressed $\mathrm{A} \beta 42$ and Dp35-HA were expressed by driving of gmr-GAL4 in fly eyes.

Acknowledgements. The authors thank Dr Miyata Yuhei for providing BG2c2 cells and Drs PP Hwang and IC Chang for assisting with electron microscopy; NHRI Optical Biology Core for confocal microscopy assistance. We also thank Dr Eric C Liebl for critical comments on the manuscript. This work was supported by the National Health Research Institutes.

1. Giannakopoulos P, Hof PR, Kovari E, Vallet PG, Herrmann FR, Bouras C. Distinct patterns of neuronal loss and Alzheimer's disease lesion distribution in elderly individuals older than 90 years. J Neuropathol Exp Neurol 1996; 55: 1210-1220.

2. Pike CJ, Burdick D, Walencewicz AJ, Glabe CG, Cotman CW. Neurodegeneration induced by beta-amyloid peptides in vitro: the role of peptide assembly state. J Neurosci 1993; 13 1676-1687.

3. Gelinas DS, DaSilva K, Fenili D, St George-Hyslop P, McLaurin J. Immunotherapy for Alzheimer's disease. Proc Natl Acad Sci USA 2004; 101 (Suppl 2): 14657-14662.

4. Lee KY, Clark AW, Rosales JL, Chapman K, Fung T, Johnston RN. Elevated neuronal Cdc2-like kinase activity in the Alzheimer disease brain. Neurosci Res 1999; 34: 21-29.

5. Noble W, Olm V, Takata K, Casey E, Mary O, Meyerson J et al. Cdk5 is a key factor in tau aggregation and tangle formation in vivo. Neuron 2003; 38: 555-565.

6. Tsai LH, Delalle I, Caviness Jr VS, Chae T, Harlow E. p35 is a neural-specific regulator subunit of cyclin-dependent kinase 5. Nature 1994; 371: 419-423.

7. Patrick GN, Zukerberg L, Nikolic M, de la Monte S, Dikkes P, Tsai LH. Conversion of p35 to p25 deregulates Cdk5 activity and promotes neurodegeneration. Nature 1999; 402 615-622.

8. Mandelkow E. Alzheimer's disease. The tangled tale of tau. Nature 1999; 402: 588-589.

9. Zukerberg LR, Patrick GN, Nikolic M, Humbert S, Wu CL, Lanier LM et al. Cables links Cdk5 and C-Abl and facilitates Cdk5 tyrosine phosphorylation, kinase upregulation, and neurite outgrowth. Neuron 2000; 26: 633-646.

10. Van Etten RA. Cycling, stressed-out and nervous: cellular functions of c-Abl. Trends $\mathrm{Cell}$ Biol 1999; 9: 179-186.

11. Koleske AJ, Gifford AM, Scott ML, Nee M, Bronson RT, Miczek KA et al. Essential roles fo the Abl and Arg tyrosine kinases in neurulation. Neuron 1998; 21: 1259-1272.

12. Hoffmann FM. Drosophila abl and genetic redundancy in signal transduction. Trends Genet 1991; 7: 351-355

13. Wang JY. Regulation of cell death by the Abl tyrosine kinase. Oncogene 2000; 19: 56435650 .

14. Glabe C. Intracellular mechanisms of amyloid accumulation and pathogenesis in Alzheimer's disease. J Mol Neurosci 2001; 17: 137-145.

15. Muqit MM, Feany MB. Modelling neurodegenerative diseases in Drosophila: a fruitful approach? Nat Rev Neurosci 2002; 3: 237-243.

16. Crowther DC, Kinghorn KJ, Miranda E, Page R, Curry JA, Duthie FA et al. Intraneuronal Abeta, non-amyloid aggregates and neurodegeneration in a Drosophila model of Alzheimer's disease. Neuroscience 2005; 132: 123-135.
17. Finelli A, Kelkar A, Song $\mathrm{HJ}$, Yang $\mathrm{H}$, Konsolaki M. A model for studying Alzheimer's Abeta42-induced toxicity in Drosophila melanogaster. Mol Cell Neurosci 2004; 26: 365-375.

18. Greeve I, Kretzschmar D, Tschape JA, Beyn A, Brellinger C, Schweizer M et al. Agedependent neurodegeneration and Alzheimer-amyloid plaque formation in transgenic Drosophila. J Neurosci 2004; 24: 3899-3906.

19. lijima K, Liu HP, Chiang AS, Hearn SA, Konsolaki M, Zhong Y. Dissecting the pathological effects of human Abeta40 and Abeta42 in Drosophila: a potential model for Alzheimer's disease. Proc Natl Acad Sci USA 2004; 101: 6623-6628.

20. Chan HY, Bonini NM. Drosophila models of human neurodegenerative disease. Cell Death Differ 2000; 7: 1075-1080.

21. Ui-Tei K, Sato S, Miyake T, Miyata Y. Induction of apoptosis in a Drosophila neuronal cell line by calcium ionophore. Neurosci Lett 1996; 203: 191-194.

22. Juang JL, Hoffmann FM. Drosophila abelson interacting protein (dAbi) is a positive regulator of abelson tyrosine kinase activity. Oncogene 1999; 18: 5138-5147.

23. Deininger $M$, Buchdunger $E$, Druker BJ. The development of imatinib as a therapeutic agent for chronic myeloid leukemia. Blood 2005; 105: 2640-2653.

24. Henkemeyer MJ, Gertler FB, Goodman W, Hoffmann FM. The Drosophila abelson protooncogene homolog: identification of mutant alleles that have pleiotropic effects late in development. Cell 1987; 51: 821-828.

25. Connell-Crowley L, Le Gall M, Vo DJ, Giniger E. The cyclin-dependent kinase Cdk5 controls multiple aspects of axon patterning in vivo. Curr Biol 2000; 10: 599-602.

26. Lee MS, Kwon YT, Li M, Peng J, Friedlander RM, Tsai LH. Neurotoxicity induces cleavage of p35 to p25 by calpain. Nature 2000; $405: 360-364$

27. Jekely $G$, Friedrich $P$. Characterization of two recombinant Drosophila calpains. CALPA and a novel homolog, CALPB. J Biol Chem 1999; 274: 23893-23900.

28. Nikolic M, Chou MM, Lu W, Mayer BJ, Tsai LH. The p35/Cdk5 kinase is a neuron-specific Rac effector that inhibits Pak1 activity. Nature 1998; 395: 194-198.

29. Tarricone C, Dhavan R, Peng J, Areces LB, Tsai LH, Musacchio A. Structure and regulation of the CDK5-p25(nck5a) complex. Mol Cell 2001; 8: 657-669.

30. Takayama N, Sato N, O'Brien SG, Ikeda Y, Okamoto S. Imatinib mesylate has limited activity against the central nervous system involvement of Philadelphia chromosomepositive acute lymphoblastic leukaemia due to poor penetration into cerebrospinal fluid. $\mathrm{Br}$ $J$ Haematol 2002; 119: 106-108.

31. Netzer WJ, Dou F, Cai D, Veach D, Jean S, Li Y et al. Gleevec inhibits beta-amyloid production but not Notch cleavage. Proc Natl Acad Sci USA 2003; 100: 12444-12449.

32. Dhavan R, Tsai LH. A decade of CDK5. Nat Rev Mol Cell Biol 2001; 2: 749-759.

33. Alvarez A, Munoz JP, Maccioni RB. A Cdk5-p35 stable complex is involved in the betaamyloid-induced deregulation of Cdk5 activity in hippocampal neurons. Exp Cell Res 2001; 264: 266-274

34. Kerokoski $P$, Suuronen $T$, Salminen A, Soininen H, Pirttila T. Cleavage of the cyclindependent kinase 5 activator p35 to p25 does not induce tau hyperphosphorylation. Biochem Biophys Res Commun 2002; 298: 693-698.

35. Saito T, Onuki R, Fujita Y, Kusakawa G, Ishiguro K, Bibb JA et al. Developmental regulation of the proteolysis of the p35 cyclin-dependent kinase 5 activator by phosphorylation. J Neurosci 2003; 23: 1189-1197.

36. Gong X, Tang X, Wiedmann M, Wang X, Peng J, Zheng D et al. Cdk5-mediated inhibition of the protective effects of transcription factor MEF2 in neurotoxicity-induced apoptosis. Neuron 2003; 38: 33-46.

37. Weishaupt JH, Neusch C, Bahr M. Cyclin-dependent kinase 5 (CDK5) and neuronal cell death. Cell Tissue Res 2003; 312: 1-8

38. Lin TY, Huang $\mathrm{CH}$, Chou WG, Juang JL. Abi enhances Abl-mediated CDC2 phosphorylation and inactivation. J Biomed Sci 2004; 11: 902-910.

39. Gertler FB, Comer AR, Juang JL, Ahern SM, Clark MJ, Liebl EC et al. enabled, a dosagesensitive suppressor of mutations in the Drosophila Abl tyrosine kinase, encodes an Abl substrate with SH3 domain-binding properties. Genes Dev 1995; 9: 521-533.

40. Rubin GM, Spradling AC. Genetic transformation of Drosophila with transposable element vectors. Science 1982; 218: 348-353. 